Please do not remove this page

RMIT

UNIVERSITY

\title{
Limit cycles in a ternary structure
}

Sadik, Amin; Hussain, Zahir

https://researchrepository.rmit.edu.au/esploro/outputs/9921862555901341/filesAndLinks?institution=61RMIT_INST\&index=null

Sadik, A., \& Hussain, Z. (2006). Limit cycles in a ternary structure. IEEE TENCON 2006, 1-4.

https://doi.org/10.1109/TENCON.2006.344168

Published Version: https://doi.org/10.1109/TENCON.2006.344168

Repository homepage: https://researchrepository.rmit.edu.au

(c) 2006 IEEE. Personal use of this material is permitted. However, permission to reprint/republish this material for advertising or promotional purposes or for creating new collective works for resale or redistribution to servers or lists, or to reuse any copyrighted component of this work in other works must be obtained from the IEEE.

Downloaded On 2023/04/26 20:00:08 +1000 


\title{
Limit Cycles in a Ternary Structure
}

\author{
Amin Z. Sadik and Zahir M. Hussain, SMIEEE \\ School of Electrical and Computer Engineering, RMIT University, Melbourne, Australia \\ amin.sadik@rmit.edu.au; zmhussain@ieee.org
}

\begin{abstract}
The limit cycle behavior of a ternary filtering structure is investigated. It is found that the limit cycles behavior is dependent on the initial conditions of quantization error and the gain parameter in addition to the level of the dc input. The system is simulated and a random search procedure is set up to explore and extract its cyclical patterns.
\end{abstract}

\section{Introduction}

One of the ill-understood behavior patterns of $\Sigma \Delta$ modulators is the generation of periodic patterns or limit cycles at its output. Such a cyclic output produces discrete noise components. Remarkable studies have been made on the limit cycle nature of the first- and second-order $\Sigma \Delta$ modulators and on their elimination [1][2][3]. Despite the numerous attempts to identify the limit cycle mode of higher order (more than 2) $\Sigma \Delta$ systems [4], an exact analysis has not been achieved yet. Understanding the limit cycle phenomenon in these systems is becoming more demanding due to their improved performance at equivalent oversampling ratios, which make them attractive to $\Sigma \Delta$ system designers. Moreover, due to their instability problem, which is the main drawback in these topologies, the limit cycle behavior should be thoroughly investigated as it has a strong relation to the issue of instability [5].

In this paper we attempted to conduct a comprehensive analysis to the ternary- $\Sigma \Delta$ topology, which is regarded as a third order $\Sigma \Delta$ modulator, both mathematically and by simulation.

\section{System Analysis}

The ternary structure in Fig.(1), which was utilized by [6][7][8][9] to design single-bit DSP systems, is basically a third-order IIR filter. To analyze this system, let as assume $x$ as the dc input, $u(k)$ is the integrator output (just precede the quantizer), and then the quantizer output, $y(k)$ will be given by: $y(k)=\operatorname{sgn}[u(k)]$.

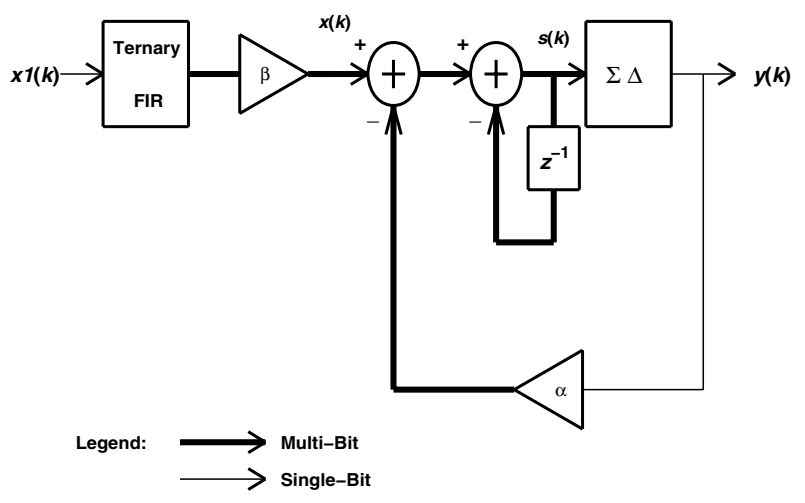

Figure 1. Single-bit ternary filter.

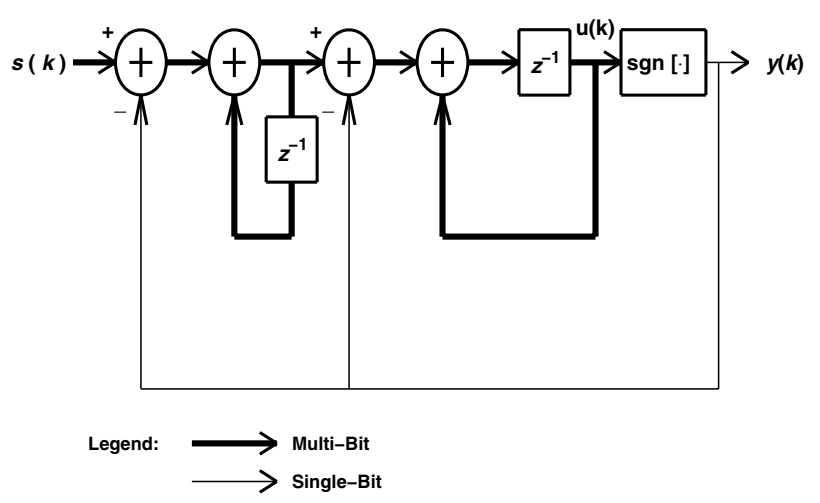

Figure 2. Single-bit second-order SD filter. 
This system can be described as follows:

$$
\begin{array}{r}
u(k)=3 u(k-1)-3 u(k-2)+u(k-3) \\
-(\alpha+2) y(k-1)+3 y(k-2)-y(k-3)+x .
\end{array}
$$

where $\alpha$ is a constant parameter. The complete iterative solution to eqn(1) is:

$$
\begin{aligned}
u(k)= & (1 / 2) k(k-1) u_{2}-k(k-2) u_{1}+p(k) u_{o} \\
& -p(k) y_{o}+k(k-2) y_{1}-(k-1) y_{2}-\alpha p(k) y_{2} \\
& -g(k, \alpha)+d(k) x .
\end{aligned}
$$

where $u_{o}, u_{1}$, and $u_{2}$ are the initial values of the integrator output, and $y_{i}=\operatorname{sgn}\left(u_{i}\right)$. The functions $p(n), g(n)$, and $d(n)$ are given as follows:

$$
\begin{aligned}
p(k) & =(k-1)(k-2) / 2 \\
g(k, \alpha) & =\sum_{n=1}^{k-3}[(1 / 2) n(n+1) \alpha+n+1] y(k-n)(4) \\
d(k) & =k p(k) / 3 .
\end{aligned}
$$

If $\alpha=1$, eqn.(2) becomes:

$u(k)=\frac{1}{2} k(k-1) e_{2}-k(k-2) e_{1}+p(k) e_{o}-\hat{g}(k)+d(k) x$.

where $e_{i}=u_{i}-\operatorname{sgn}\left(u_{i}\right)$, whereas $\hat{g}(k)$ represents the case when $\alpha=1$ and is given by:

$$
\hat{g}(k)=g(k, 1)=\frac{1}{2} \sum_{n=1}^{k-3}[(n+2)(n+1)] y(k-n) .
$$

If we assume a limit cycle of length $L$ has occurred in the system, then from eqn.(2), $y(k+L)=y(k)$. Solving and arranging for $L$ yields:

$$
\begin{aligned}
& -\frac{L^{2}}{2}\left(u_{2}-\alpha y_{2}-2 e_{1}+e_{o}\right) \\
& +\frac{L}{2}\left(u_{2}+(2-3 \alpha) y_{2}-4 e_{1}+3 e_{o}\right) \\
& -k L\left(u_{2}-\alpha y_{2}-2 e_{1}+e_{o}\right)-(g(k, \alpha)-g(k+L, \alpha)) \\
& =(d(k+L)-d(k)) x .
\end{aligned}
$$

If $\alpha=1$, then $u_{2}-\alpha y_{2}=u_{2}+(2-3 \alpha) y_{2}=e_{2}$, and eqn.(8) becomes:

$$
\begin{gathered}
-\frac{L^{2}}{2}\left(e_{2}-2 e_{1}+e_{o}\right)+\frac{L}{2}\left(e_{2}-4 e_{1}+3 e_{o}\right) \\
-k L\left(e_{2}-2 e_{1}+e_{o}\right)-\hat{g}(k)-\hat{g}(k+L) \\
=(d(k+L)-d(k)) x .
\end{gathered}
$$

It is evident from eqn.(8) that the gain parameter has an important role in this matter, as it affects the initial conditions as well as the steady state operation. Both eqn.(8) and eqn.(9) imply that the occurrence of limit cycles in the this structure is conditional. If we adopt the approach in [10][11], by assuming $x$ to belong to the set of rational numbers $a / b$, where $a$ and $b$ are prime integers, then all the terms that contain the signum function and multiplied by integer factors are also a subset of the same set of rational numbers, $a / b$. However, this condition is not sufficient in our structure to produce limit cycles and another condition should be met as well. Specifically, in eqn.(8) the factor $\left(u_{2}-\alpha y_{2}-2 e_{1}+e_{o}\right)$ or the factor $\left(e_{2}-2 e_{1}+e_{o}\right)$ in eqn.(9) must be equal to zero. Therefore, limit cycles occur in the ternary- $\Sigma \Delta$ system with periods of $L$ given by:

$$
L=\frac{\left(b_{k+L}-b_{k}\right)}{a\left(u_{2}-(2-3 \alpha) y_{2}-4 u_{1}+3 u_{o}\right)} .
$$

\section{Limit Cycles: Analysis and Simulation}

In this section we focus on the detection and extraction of the limit cycles at the system output. This is done in both the frequency domain (using FFT) and time domain (using autocorrelation). The effect of OSR (oversampling ratio) will also be considered.

The cyclic sequences will be described as follows [12]:

$$
Q_{(i), j}=\left[+q_{1},-q_{1}, \ldots+q_{i-1},-q_{i-1},+q_{i},-q_{i}\right]
$$

where $i$ denotes the number of transitions from +1 to -1 (or -1 to +1 ) within the limit cycle period, while the subscript $j$ represents any integer. The values between brackets represent successive outputs that constitute one cycle, where $+q_{i}$ represents the number of consecutive +1 's, whereas $-q_{i}$ represents the number of consecutive -1 's, both at the $i^{\text {th }}$ transition.

\subsection{Zero-Input Limit Cycles}

Fig.(3) illustrates an approach to find periodic patterns in the ternary structure. A sufficiently large number of lags (clock periods) in the autocorrelation function, $\mathrm{R}_{\mathrm{yy}}(n)$, should be used to insure that no longer cyclic period does exist. The figure reveals that the maximum limit cycle length $L_{\max }$ for zero input in the ternary- $\Sigma \Delta$ topology shown in Fig.(1) is $L_{\max }=8$. Same result can be obtained from the frequency domain, whereby the fundamental limit cycle frequency $f_{o}$ and its harmonics are located and then $L_{\max }$ can be calculated as follows:

$$
L_{\max }=f_{s} / f_{o}
$$

where $f_{s}$ is the sampling frequency. However, one should be aware that this is true only for stationary signals. Furthermore, the number of transitions $i$ within the limit cycle is calculated as shown in Fig.(4), where it is obvious that 


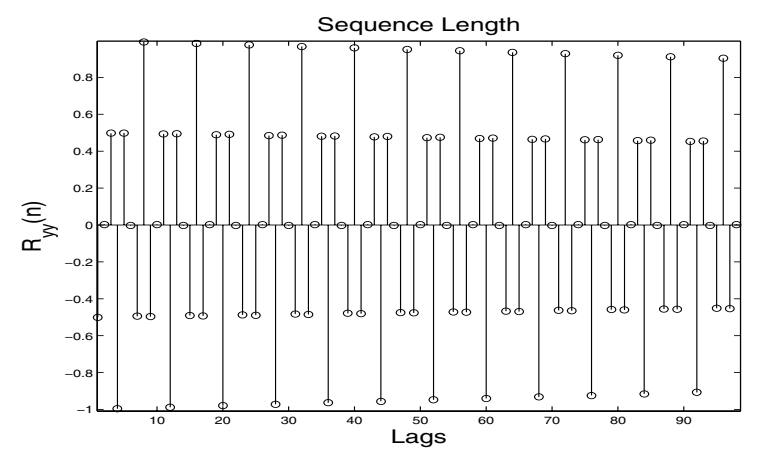

Figure 3. Fundamental limit cycle in the autocorrelation function for $\mathrm{x}=\mathbf{0}$.

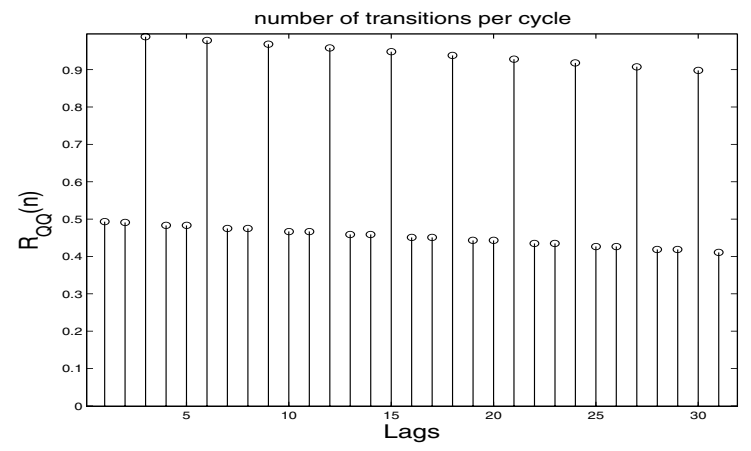

Figure 4. Number of transitions in the fundamental cycle of autocorrelation for $\mathrm{x}=0$.

$i=3$ in that case, with the main sequence of the limit cycles as:

$$
Q_{(3), 1}=\left[2_{+}, 1_{-}, 1_{+}, 2_{-}, 1_{+}, 1_{-}\right] .
$$

The constant parameter $\alpha$ has a vital role on the limit cycle behavior of the system under investigation in the case of zero-input.

Fig. (5) shows the phase plane of the limit cycle that the ternary structure traps in (after a sufficiently long time)for $x=0, \alpha=0.1$ and initial conditions $u_{o}=0.5, u_{1}=$ $0.7, u_{2}=0.8$.

It is worth noting here that the difference between the average output of any complete limit cycle and the input is zero, i.e.,

$$
x-\frac{1}{L} \sum_{k}^{k+L} y(k)=0
$$

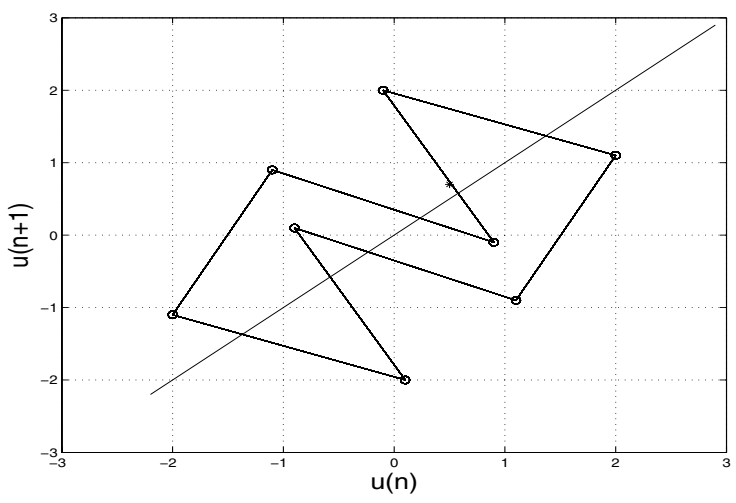

Figure 5. Phase portrait $u_{o}=0.5, u_{1}=$ $0.7, u_{2}=0.8, \alpha=0.1$ for $\mathbf{x}=\mathbf{0}$.

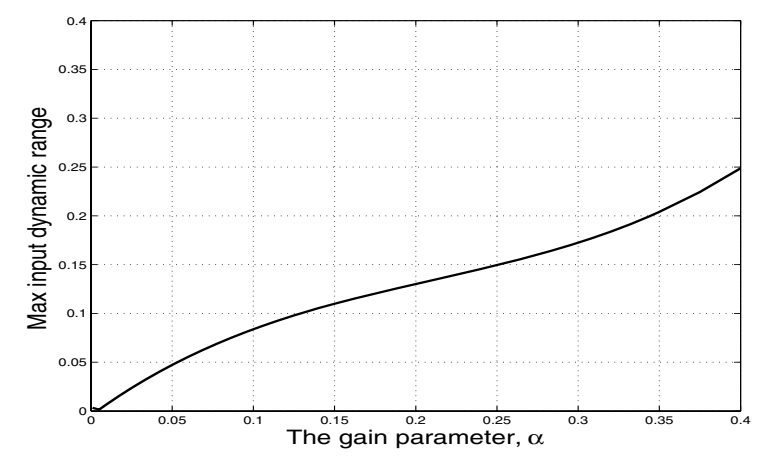

Figure 6. Gain vs. maximum dc input beyond which no limit cycle occurs.

\subsection{Limit Cycles for DC Inputs}

The ternary- $\Sigma \Delta$ filter exhibits a highly non-linear limit cycle behavior. Furthermore, the parameter $\alpha$ appends an extra variable to the problem. On one hand, $\alpha$ controls the maximum dc input $x_{\max }$ beyond which no limit cycle can be detected. This can be seen in Fig.(6). On the other hand, as anticipated by eqn.(8), $\alpha$ may alter the limit cycle behavior through the variation of both the initial and steady-state conditions of the system. This alteration extends to include the quantization noise structure as well.

The frequencies of these patterns normally reside in the baseband region, however, their power is relatively low. This is due to the noise shaping effect of the $\Sigma \Delta$ modulator in this band of frequency. Shorter cycles that are in fact subsets of $L_{\max }$ (and consequently located in a higher frequency band) suffer several orders of magnitude less attenuation, and therefore aggravate the problem of instability. As an example in this simulation: at $x=1 / 20$, Fig.(7) depicts the 


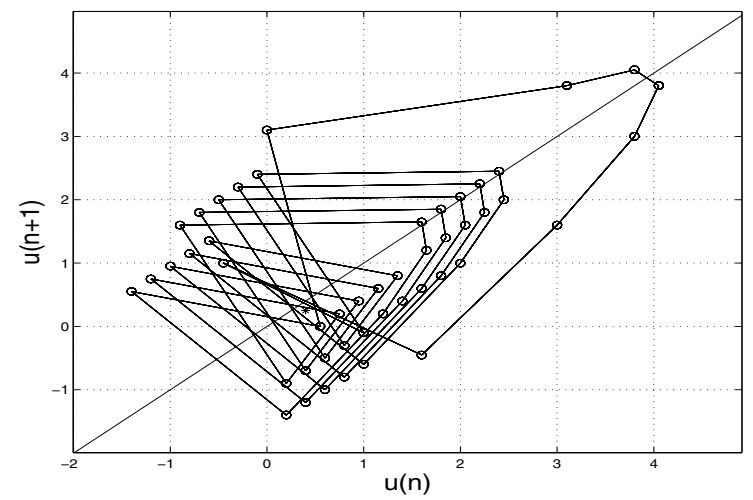

Figure 7. Phase plane of limit cycle for $u_{O}=$ $0.4, u_{1}=0.25, u_{2}=0, \alpha=0.1$ and $x=1 / 20$.

phase plane of limit cycle with $L_{\max }=48$, and the number of transitions $=12$, the available limit cycle sequences are: $Q_{(12), 1}=\left[6_{+}, 1_{-}, 1_{+}, 1_{-}, 4_{+}, 1_{-}, 2_{+}, 1_{-}, 4_{+}, 1_{-}, 2_{+}, 1_{-}\right.$, $\left.4_{+}, 1_{-}, 2_{+}, 1_{-}, 4_{+}, 1_{-}, 2_{+}, 1_{-}, 4_{+}, 1_{-}, 1_{+}, 1_{-}\right]$ $Q_{(2), 1}=\left[2_{+}, 1_{-}, 4_{+}, 1_{-}\right] ; Q_{(2), 2}=\left[4_{+}, 1_{-}, 2_{+}, 1_{-}\right] ;$etc.

It can be easily seen that $Q_{(2), 1}$ is a subset of the mother sequence $Q_{(12), 1}$ and $Q_{(2), 2}$ is not valid as it is a shifted version of $Q_{(12), 1}$. Our findings are as follows:

* The structure may have long limit cycles (e.g., $\left.L_{\max }=200,300, \ldots\right)$, and subsets of shorter cycles are given by $L=L_{\max } / m, \quad m=1,2, \cdots$.

* The relation between the integers $a, b(x=a / b)$ and $L$ (e.g., $L=a+b$ and $f_{o}=[m / b] f_{s}$ ), well-known in the firstand second-order $\Sigma \Delta$, is quite valid in this ternary structure, which is basically a third-order $\Sigma \Delta$.

$\star$ Although it is difficult to predict $L_{\max }$ for a given input, it seems that a certain qualitative periodicity does exist.

\section{Conclusions}

A ternary- $\Sigma \Delta$ structure was analyzed mathematically without imposing any approximations. It was evident that the system exhibits a conditional limit cycle behavior. These conditions includes the initial quantization noise conditions and the constant gain parameter in addition to the dc input magnitude. The system was then simulated extensively and a random search method is utilized to discover and extract the limit cycles and identify their features. It seemed that this topology, which is similar to third order $\Sigma \Delta$ modulator possesses a highly non-linear behavior and is similar in some aspects to that of the first and second order $\Sigma \Delta$ modulators. Further insight investigation is recommended, as the issue of limit cycles in higher order modulators is vital for instability problem.

\section{References}

[1] F. Dachselt, and S. Quitzk, "Structure and information content in sequences from the single-loop sigma-delta modulator with dc input," Preceedings of 2004 IEEE International Symposium on Circuits and Systems (ISCAS'04), pp. IV-685-688, May 2004.

[2] W. Chou and R. M. Gray, "Dithering and its effects on sigmadelta and multistage sigma-delta modulation," IEEE Transactions on Information Theory, vol. 37, no. 3, pp. 500-513, May 1991.

[3] R. Shreier, "Destabilizing limit cycles in delta-segma modulators with chaos," IEEE International Symposium on Circuits and Systems (ISCAS '93), vol. 2, pp. 1369-1372, May 1993.

[4] P. Steiner and W. Yang, "A framework for analysis of higherorder sigma-delta modulators," IEE Electronics Letters, vol. 44, no. 1, pp. 1-10, Jan. 1997.

[5] N. Wong and T.-S. Ng, "Fast detection of instability in sigmadelta modulators based on unsatable embedded limit cycles," IEEE Transaction on Circuit and Systems-II: Express Briefs, vol. 51, no. 8, pp. 442-449, Aug. 2004.

[6] A. C. Thompson, Z. M. Hussain, and P. O'Shea, "Efficient digital single-bit resonator," IEE Electronics Letters, vol. 40, no. 2, Feb. 2003.

[7] A. C. Thompson, P. O'Shea, Z. M. Hussain, and B. R. Steele, "Efficient single-bit ternary digital filtering using sigma-delta modulator," IEEE Signal Processing Letters, vol. 11, no. 2, pp. 162-166, Feb. 2004.

[8] A. Z. Sadik, Z. M. Hussain, and P. O'Shea, "Structures for Single-Bit Digital Comb Filtering," Proceeedings of the 2005 Asian Pacific Conference on Communications (APCC 2005), pp. 545-548, Perth, Australia, Oct. 2005.

[9] A. Z. Sadik, Z. M. Hussain, and P. O'Shea, "A Single-Bit Digital DC-Blocker Using Ternary Filtering," IEEE TENCON 2005, pp. 1793-1798, Melbourne, Australia, Nov. 2005.

[10] V. Friedman, "The structure of the limit cycles in sigma delta modulation," IEEE Transaction on Communications, vol. 36, no. 8, pp. 972-979, Aug. 1988.

[11] R. M. Gray, "Spectral analysis of quantization noise in a single-loop sigma-delta modulator with dc input," IEEE Transaction on Communications, vol. 37, no. 6, pp. 588-599, June 1989.

[12] D. Hyun and G. Fischer, "Limit cycles and pattern noise in single-stage single-bit delta-sigma modulators," IEEE Transaction on Circuit and Systems-I:Fundamental Theory and Applications, vol. 49, no. 5, pp. 646-656, May 2002. 